\title{
A Simple CRLH LWA Circuit Condition for Constant Radiation Rate
}

\author{
J. S. Gomez-Diaz ${ }^{1}$, Student Member, IEEE, D. Cañete-Rebenaque ${ }^{1}$, \\ and A. Alvarez-Melcon ${ }^{1}$, Senior Member, IEEE
}

\begin{abstract}
A simple analytical condition for the radiation resistance and conductance $(R, G)$ of an equivalent composite right/left-handed (CRLH) balanced leaky-wave antenna unit-cell is proposed to achieve a constant radiation rate. Similar to the CRLH "balanced condition", which allows a smooth phase transition from the left-handed to the right-handed frequency regions, the proposed $R-G$ condition provides a continuous and smooth transition of the radiation losses from the left-handed to the right-handed frequency region. Besides, this condition also solves the phase fluctuations which traditionally occur around the CRLH transition frequency, due to real radiation losses. Several numerical results are included to confirm the uniform radiation rate obtained when the new derived condition is used.
\end{abstract}

Index Terms-Composite Right/Left-Handed (CRLH) LeakyWave Antennas (LWAs), Broadside Radiation.

\section{INTRODUCTION}

Composite right/left-handed leaky-wave antennas (CRLH LWAs) [1], [2] introduced full-space continuous beam scanning, from backfire to endfire, including the broadside direction. Among other possibilities, these antennas can be fabricated in microstrip (see Fig. 3.39 of [1] or Fig. 1 of [3]), or coplanar (see Fig. XX of [2]) technologies. The analysis of CRLH LWAs has usually been performed using equivalent circuit models [1], [2], which pay special attention to the phase constant $[\beta(\omega)]$, allowing to obtain "balanced" designs (able to radiate at the broadside direction). However, radiation losses are usually not taken into account in this type of models.

Recently, there has been a lot of interest in obtaining a constant radiation rate for all beam directions [3], [4], which allows a beam to scan from backfire to endfire without a decrease of efficiency at any particular direction. In this context, we propose here a new analytical condition for the equivalent radiation resistors $(R, G)$ of the unit-cell model, which forces the CRLH LWA to radiate with the same radiation rate towards any direction in space, with the exception of those close to the backfire and endfire directions. The proposed $R-G$ condition may be considered as an optimization of the CRLH "balanced condition" [1], as concerns to the radiation losses, which allows a continuous and smooth transition of the radiation losses from the left-handed to the right-handed frequency region. Besides, we show that the use of the novel condition completely removes the phase constant fluctuations around the transition frequency which appear due to real radiation losses, leading to more stable unit-cell designs.

\footnotetext{
${ }^{1}$ Technical University of Cartagena, Campus Muralla del Mar s/n, Cartagena, E-30202, Spain, E-mail: jsebastian_gomez@ono.com
}

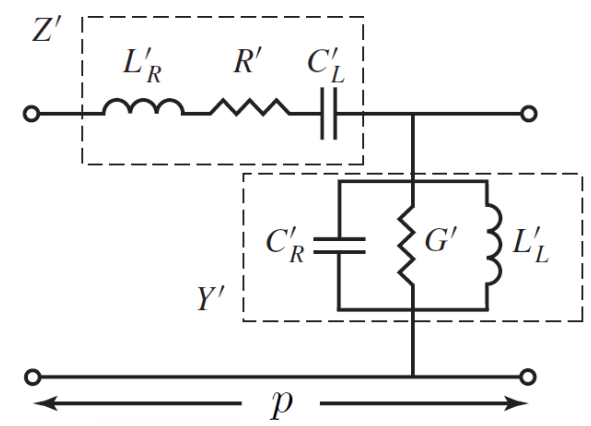

Fig. 1: Equivalent unit-cell model of a CRLH transmission line, which operates as a leaky-wave antenna. The series resistance $\left(R^{\prime}\right)$ and the shunt conductance $\left(G^{\prime}\right)$ provide the radiation losses of the antenna. Dielectric and ohmic losses are neglected for simplicity.

Finally, to demonstrate the usefulness of the developed condition, the radiation losses from two different CRLH LWAs taken from the literature are carefully analyzed. Numerical simulations completely demonstrate that an adequate combination of the $R$ and $G$ resistor values is required to obtain a constant radiation rate in the whole space, avoiding any decrease of the radiation efficiency at the broadside direction.

\section{DERIVING AN $R-G$ CONDITION FOR CONSTANT Full-SPACE RADIATION RATE}

The unit-cell model related to a CRLH transmission line, which operates as a leaky-wave antenna, is shown in Fig. 1 [1]. It consists of a per unit-length impedance (Z') and a perunit length admittance ( $\left.Y^{\prime}\right)$, which may be expressed as

$$
\begin{aligned}
Z^{\prime} & =R^{\prime}+j \omega L_{R}^{\prime}+\frac{1}{j \omega C_{L}^{\prime}}, \\
Y^{\prime} & =G^{\prime}+j \omega C_{L}^{\prime}+\frac{1}{j \omega L_{R}^{\prime}},
\end{aligned}
$$

following the notation introduced in [1]. Since dielectric and ohmic losses are neglected, the series and shunt resistors represent the radiation losses of the LWA. Besides, note that all variables are normalized with respect to the unit-cell length $(p)$, as in [1]. In this model $R^{\prime}$ and $G^{\prime}$ are assumed to be constant with frequency. This is an approximation which is only valid around the central wide-band of the fast-wave frequency region, but it is not accurate close to the fast-wave frequency region borders, where, physically, $R^{\prime}$ and $G^{\prime}$ may present important variations as a function of frequency. 
Let us assume that the homogenous condition $\left(p \ll \lambda_{g}\right)$ is satisfied (where $\lambda_{g}$ is the guided wavelength). In the limiting case $(p \rightarrow 0)$, the complex propagation constant associated to this unit-cell placed in an infinite environment reads [1]

$$
\gamma=\alpha+j \beta=\sqrt{Z^{\prime} Y^{\prime}} .
$$

Furthermore, let us assume that the CRLH "balance condition" is satisfied [1]. This condition implies the mutual cancelation of the series and shunt resonances, and it may be expressed as

$$
C_{L}^{\prime} L_{R}^{\prime}=C_{R}^{\prime} L_{L}^{\prime}
$$

After some tedious but straightforward manipulations, the phase $[\beta(\omega)]$ and attenuation $[\alpha(\omega)]$ constants may be expressed, for the unit cell of Fig. 1, as shown in Eq. (5) and Eq. (6) [shown at the top of the next page]. For the sake of compactness, we have introduced in these expressions the variables $\omega_{0}$ (transition frequency, related to broadside radiation) and $\omega_{L}$, which are defined as

$$
\omega_{0}^{\prime}=\frac{1}{\sqrt[4]{C_{L}^{\prime} L_{L}^{\prime} C_{R}^{\prime} L_{R}^{\prime}}}, \quad \omega_{L}^{\prime}=\frac{1}{\sqrt{C_{L}^{\prime} L_{L}^{\prime}}} .
$$

As an illustrative example, consider a CRLH LWA unit-cell with circuital parameters of $C_{R}=C_{L}=1.0 \mathrm{pF}, L_{R}=L_{L}=$ $2.5 \mathrm{nH}, R=5 \Omega, G=0.04 \Omega^{-1}$ and a unit-cell length of $p=1.5 \mathrm{~cm}$. Note that the radiation losses of this antenna may be important and they can not be neglected (as usually occurs with other circuital models used in the past [1]).

Fig. 2 presents the dispersion diagram $[\beta(\omega)]$ for this example. As can be seen in the figure, there are some fluctuations in the phase constant around the transition frequency (even though that the line is balanced). This is due to the effect of real radiation losses, which can not be neglected anymore. Besides, note that the phase constant lies within the fast-wave region as long as frequency increases, which means that this antenna continues to radiate at very high frequencies. In Fig. 3 (solid line), the radiation losses $[\alpha(\omega)]$ related to this unitcell are shown. As can be seen in the figure, they present a discontinuity at $\omega_{B F}$ (which denotes the initial point of the fast-wave region). This non-physical behavior appears because the CRLH LWA circuital-model of Fig. 1 is an approximation, which consider that $R^{\prime}$ and $G^{\prime}$ are frequency-independent. Specifically, they have a strictly zero value outside of the fast-wave region (no radiation) but a constant and welldefined value inside that region (radiation). However, in a real structure, the values of $R^{\prime}$ and $G^{\prime}$ are frequency-dependent, and, after some possible fluctuations, they must gradually tend to zero at the fast-wave frequency region edges. Furthermore, as shown in Fig. 3, the radiation losses suffers an important decrease at the transition frequency $\left(\omega_{0}\right)$. This effect implies that the radiation from backfire to endfire is not constant, and it is usually reduced at the broadside direction (see [3], [4]). Besides, it is interesting to note that radiation losses rapidly tends to a finite and well-defined value as long as the frequency is far away from $\omega_{0}$.

From Eq. (6), it is simple to demonstrate that the value of the radiation losses at the transition frequency $\left(\omega_{0}\right)$ is [3]

$$
\alpha\left(\omega_{0}\right)=\sqrt{R^{\prime} G^{\prime}} .
$$

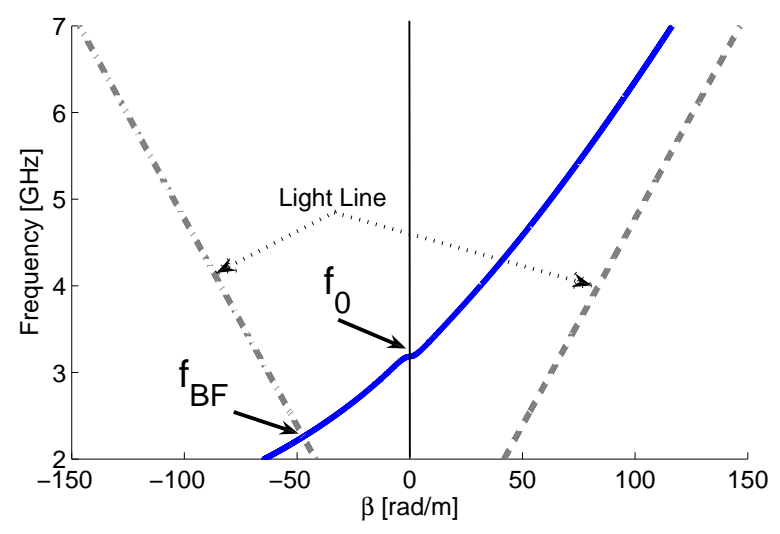

Fig. 2: Dispersion diagram computed by Eq. (5) associated to a single CRLH LWA unit-cell. The circuital parameters are $C_{R}=C_{L}=1.0 \mathrm{pF}, L_{R}=L_{L}=2.5 \mathrm{nH}, R=5 \Omega, G=$ $0.04 \Omega^{-1}$ and the unit-cell length is $p=1.5 \mathrm{~cm}$.

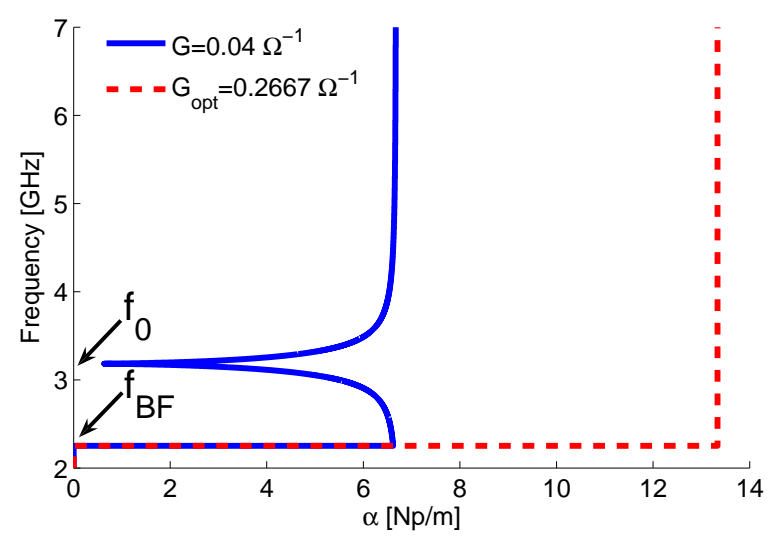

Fig. 3: Radiation losses diagram computed by Eq. (6) associated to a single CRLH LWA unit-cell. The circuital parameters are the same as in Fig. 2. The values of the shunt conductance, $\mathrm{G}$, are $0.04 \Omega^{-1}$ (solid line) and $0.2667 \Omega^{-1}$ (dashed line, optimized result).

Besides, the value of the radiation losses at a frequency different of $\omega_{0}$ (in the limiting case, $\omega \rightarrow \infty$ ) may be expressed as

$$
\lim _{\omega \rightarrow \infty} \alpha(\omega)=\sqrt{\frac{R^{\prime} G^{\prime}}{2}+\omega_{0}^{2} \frac{R^{\prime 2} C_{L}^{\prime} C_{R}^{\prime}+G^{\prime 2} L_{L}^{\prime} L_{R}^{\prime}}{4}} .
$$

In order to obtain a constant radiation rate, the radiation at the broadside direction must be equal to the radiation outside of this direction. In the limiting case, this condition may be expressed as

$$
\alpha\left(\omega_{0}\right)=\lim _{\omega \rightarrow \infty} \alpha(\omega) .
$$

After some straightforward manipulations, Eq. (10) may be reduced to the following simple condition

$$
\frac{R^{\prime}}{G^{\prime}}=\sqrt{\frac{L_{L}^{\prime} L_{R}^{\prime}}{C_{L}^{\prime} C_{R}^{\prime}} .}
$$




$$
\begin{aligned}
& \beta(\omega)= \pm \sqrt{\frac{\left[\left(\frac{\omega}{\omega_{0}^{\prime}}\right)^{2}-1\right]^{2}-R^{\prime} G^{\prime}\left(\frac{\omega}{\omega_{L}^{\prime}}\right)^{2}+\sqrt{\left[\left(\frac{\omega}{\omega_{0}^{\prime}}\right)^{4}+\left(\frac{\omega}{\omega_{0}^{\prime}}\right)^{2}\left(G^{\prime 2} \frac{L_{L}^{\prime}}{C_{R}^{\prime}}-2\right)+1\right]\left[\left(\frac{\omega}{\omega_{0}^{\prime}}\right)^{4}+\left(\frac{\omega}{\omega_{0}^{\prime}}\right)^{2}\left(R^{\prime 2} \frac{C_{L}^{\prime}}{L_{R}^{\prime}}-2\right)+1\right]}}{2\left(\frac{\omega}{\omega_{L}^{\prime}}\right)^{2}}}, \\
& \alpha(\omega)=\sqrt{\frac{-\left[\left(\frac{\omega}{\omega_{0}^{\prime}}\right)^{2}-1\right]^{2}+R^{\prime} G^{\prime}\left(\frac{\omega}{\omega_{L}^{\prime}}\right)^{2}+\sqrt{\left[\left(\frac{\omega}{\omega_{0}^{\prime}}\right)^{4}+\left(\frac{\omega}{\omega_{0}^{\prime}}\right)^{2}\left(G^{\prime 2} \frac{L_{L}^{\prime}}{C_{R}^{\prime}}-2\right)+1\right]\left[\left(\frac{\omega}{\omega_{0}^{\prime}}\right)^{4}+\left(\frac{\omega}{\omega_{0}^{\prime}}\right)^{2}\left(R^{\prime 2} \frac{C_{L}^{\prime}}{L_{R}^{\prime}}-2\right)+1\right]}}{2\left(\frac{\omega}{\omega_{L}^{\prime}}\right)^{2}}} .
\end{aligned}
$$

The importance of the proposed $R-G$ condition is two fold. First, this condition forces the CRLH LWA to radiate with the same radiation rate in the whole space (from backfire to endfire), without any decrease at the broadside direction. Physically, this is because the radiated field is a combination from the series and shunt currents along the CRLH LWA unit-cell. This is demonstrated in Fig. 3 (dashed line), where the radiation losses related to the unit-cell model (using the previous circuital parameters) are recomputed employing an optimized value of $G$ [which satisfies Eq. (11)]. As can be seen in the figure, radiation losses are constant for all frequencies (inside the fast-wave region). However, note that this circuital model is an approximation, and it only represents a physical structure on these frequencies which are not close to the fast-wave region borders. Second, the phase constant is optimized at the transition frequency, solving the fluctuations introduced by real radiation losses. This is demonstrated in Fig. 4, which presents a detailed zoom of the dispersion diagram at the transition frequency. As can be seen in the figure, the use of non-optimum values of $R$ and $G$ (solid line) leads to phase fluctuations around the transition frequency, which deteriorates the broadside radiation. On the other hand, the use of optimized $R$ and $G$ values (dashed line) completely removes these fluctuations around the transition frequency, and allows a perfect radiation at the broadside direction.

In order to make a real design of a CRLH LWA unitcell, the series $\left(R^{\prime}\right)$ and shunt $\left(G^{\prime}\right)$ per-unit-cell resistors must be carefully considered. Unfortunately, to the best of our knowledge, there are not CAD expressions which easily provide the parameters $R^{\prime}$ and $G^{\prime}$. Therefore, as in the case of regular CRLH LWA design, the use of full-wave software is still required to obtain and optimize these values (see [3]). However, thanks to the proposed $R^{\prime} / G^{\prime}$ condition, the optimization process is simplified, fixing a very clear goal. Besides, this condition allows the designer to directly control the total amount of radiation losses (modifying the values of $R^{\prime}$ and $G^{\prime}$ ) while obtaining a more stable CRLH LWA unitcell design, solving the phase fluctuation around the transition frequency and maintaining the radiation rate constant in a wide frequency band (ratio between $R$ and $G$ satisfied).

\section{NUMERICAL VALIDATION}

In this section, the radiation losses from two different CRLH LWAs taken from the literature are carefully analyzed. Next, the derived $R-G$ condition is applied in each case to obtain the resistance $\left(R^{\prime}\right)$ and conductance $\left(G^{\prime}\right)$ values which model the CRLH LWA unit-cell. It is then demonstrated that the computed radiation losses i) are completely constant with

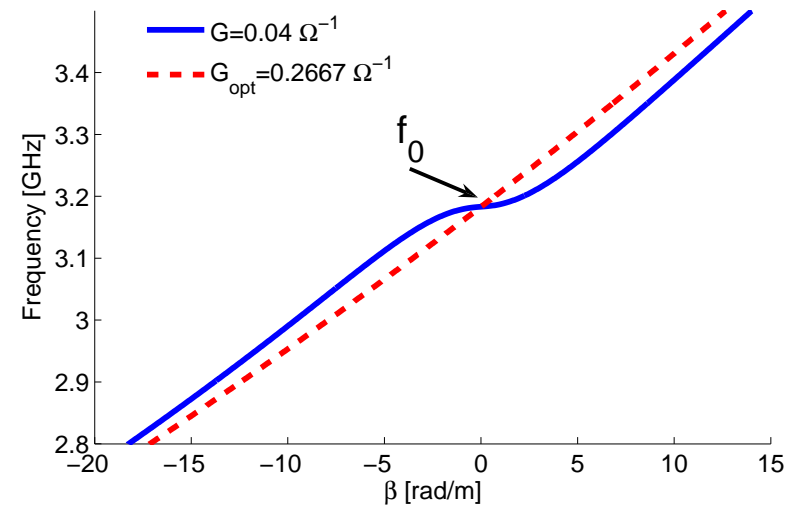

Fig. 4: Detail of the dispersion diagram around the transition frequency $\omega_{0}$ associated to a single CRLH LWA unit-cell. The circuital parameters are the same as in Fig. 2. The values of the shunt conductance, G, are $0.04 \Omega^{-1}$ (solid line) and $0.2667 \Omega^{-1}$ (dashed line, optimized result).

frequency (except at the fast-wave region borders), ii) do not show any decrease of the radiation efficiency at the broadside direction and iii) agree very well against full-wave simulation and measurements.

Let us examine the work presented in [3], where a broadside optimization approach of CRLH LWAs based on interdigital capacitors and planar stubs is introduced. Specifically, a parametric full-wave study is presented as a function of the stubs length, achieving an almost constant radiation losses for all beam directions, including broadside. In this case, the derived circuital parameters of the unit-cell were $C_{R}=1.47 \mathrm{pF}$, $C_{L}=0.6 \mathrm{pF}, L_{R}=2.09 \mathrm{nH}, L_{L}=0.85 \mathrm{nH}, R=1.18 \Omega$, $G=0.4 \times 10^{-3} \Omega^{-1}$, with a unit-cell length of $p=6 \mathrm{~mm}$. The normalized radiation losses versus frequency for this example, computed using a Bloch-wave approach [1] [5] are shown in Fig. 5 (solid line). This graph reproduces the results presented in [3]. As can be seen in the figure, radiation losses are not completely linear as a function of frequency, and some small variations appear around the transition frequency. This is because the $R-G$ values do not satisfy Eq. (11). However, these values are close to the optimum condition. This explains that the optimized antenna of [3] presents an almost constant full-space radiation rate. Let us keep the $R$ value constant, and modify $G$ towards its optimum value $\left(G=0.831 \times 10^{-3} \Omega^{-1}\right.$ ). The radiation losses in this case are also shown in Fig. 5 (dashed line). As expected, they are totally linear as a function of frequency, leading to a radiation with constant radiation 


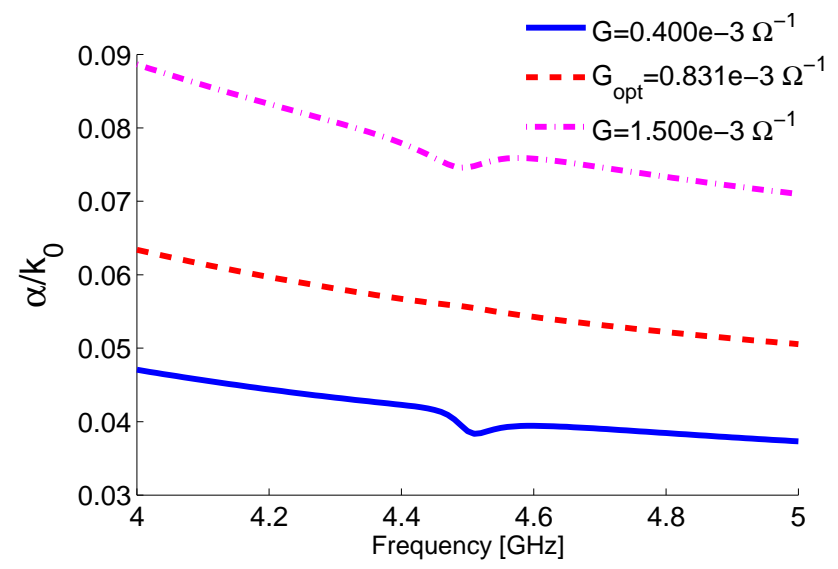

Fig. 5: Normalized attenuation constant $\left[\alpha(\omega) / k_{0}\right]$ obtained with a Bloch-wave analysis [1] [5]. The circuital parameters (from [3]) are $C_{R}=1.47 \mathrm{pF}, C_{L}=0.6 \mathrm{pF}, L_{R}=2.09 \mathrm{nH}$, $L_{L}=0.85 \mathrm{nH}, R=1.18 \Omega$, and the unit-cell length is $p=$ $6 \mathrm{~mm}$. The values of the shunt conductance, $\mathrm{G}$, are $0.4 \mathrm{~m} \Omega^{-1}$ (solid line, same result as in [3]), $0.831 \mathrm{~m} \Omega^{-1}$ (dashed line, optimized result) and $1.5 \mathrm{~m} \Omega^{-1}$ (dashed-dotted line).

rate. This result fully demonstrates the usefulness of the new proposed $R-G$ condition. Finally, if we keep increasing the value of $G$ (for instance, $G=1.5 \times 10^{-3} \Omega^{-1}$ ), the optimum $R-G$ condition is not satisfied anymore, and the radiation losses suffer again from variations around the transition frequency (see Fig. 5, dashed-dotted line).

Let us now consider the CRLH LW antenna presented in [6]. The line is composed of $24-6.1 \mathrm{~mm}$ long- unit-cells, with circuital parameters of $C_{R}=0.5 \mathrm{pF}, C_{L}=0.68 \mathrm{pF}$, $L_{R}=2.45 \mathrm{nH}$ and $L_{L}=3.35 \mathrm{nH}$. The measured radiation losses [6], $\alpha(\omega)$, are shown in Fig. 6 (black '*'). In order to model these radiation losses, the value of $R$ may be modified in order to fit with measurements, keeping the conductance $G$ equal to 0 (this is the approach followed in [6]). A good possibility to fit with the measurement data is to use $R=$ $3.1 \Omega$. The radiated losses computed with these values are also depicted in Fig. 6 (dashed-line). For this computation, a transmission line (ABCD) matrix approach, combined with the Floquet's theorem, has been applied [6]. As can be seen in the figure, the agreement between simulations and measurements is good in the whole frequency region, with the exception of the frequencies around the transition frequency $\left(\omega_{0}\right)$. This is because this model only takes into account the influence of $R$, whereas the radiation at the broadside direction requires from both, the series and the shunt resistors [ $R$ and $G$, see Eq. (8)]. This is further confirmed from the $\alpha(\omega)$-measurements, which do not tend to 0 around the transition frequency, since in the real structure $G$ is close to its optimum value.

At this point, the values of $R$ and $G$ are modified in order to optimize the radiation at the broadside direction. Therefore, the $R-G$ ratio condition introduced in Eq. (11) is applied. The derived $R$ and $G$ parameters are $R=1.55 \Omega$ and $G=0.3155 \mathrm{~m} \Omega^{-1}$. The computed radiation losses, using these parameters, are shown in Fig. 6 (solid line). As can be seen in the figure, the agreement of the proposed model

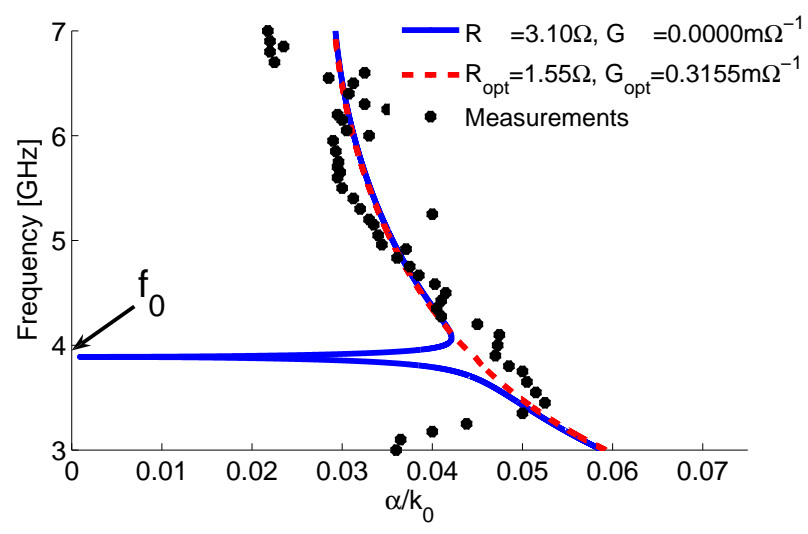

Fig. 6: Measured attenuation constant $\left[\alpha(\omega) / k_{0}\right]$ obtained from the $24-6.1 \mathrm{~mm}$ long- unit-cells CRLH LWA prototype presented in [6]. The circuital parameters of the line are $C_{R}=$ $0.5 \mathrm{pF}, C_{L}=0.68 \mathrm{pF}, L_{R}=2.45 \mathrm{nH}$ and $L_{L}=3.35 \mathrm{nH}$. Simulation results, from a Bloch-wave analysis method, are shown for the case of $R=3.10 \Omega$ and $G=0.0 \mathrm{~m} \Omega^{-1}$ (dashed line), and for the case of $R=1.55 \Omega$ and $G=0.3155 \mathrm{~m} \Omega^{-1}$ (solid line, optimized result).

with respect to measurement is good in the whole frequency range, including the broadside direction. In fact, there is not any decrease of the radiation losses at the transition frequency, as occurs with the measurement data. This result demonstrates that the derived $R-G$ condition is indeed required to obtain a constant radiation losses, including the radiation at the broadside direction.

\section{CONCLUSIONS}

A simple circuit condition has been proposed to achieve constant radiation rate from a balanced CRLH LWA unit-cell. It has been demonstrated that the proposed condition solves the phase fluctuations which occurs close to the CRLH transition frequency, due to real radiation losses. Thereby, the novel circuital condition provides a more stable CRLH LWA unitcell design, allowing a continuous and smooth transition of the radiation losses from the left-handed to the right-handed frequency region.

\section{REFERENCES}

[1] C. Caloz and T. Itoh, Electromagnetic Metamaterials: Transmission Line Theory and Microwave Applications. Wiley and IEEE Press, 2005.

[2] G. V. Eleftheriades and K. G. Balmain, Eds., Negative-Refraction Metamaterials: Fundamental Principles and Applications. Hoboken, NJ: Wiley \& IEEE Press, 2005.

[3] S. Paulotto, P. Baccarelli, F. Frezza, and D. R. Jackson, "Full-wave modal dispersion analysis and broadside optimization for a class of microstrip crlh leaky-wave antennas," IEEE Transactions on Microwave Theory and Techniques, vol. 56, no. 12, pp. 2826 - 2837, December 2008.

[4] _ "A novel technique for open-stopband suppression in 1-d periodic printed leaky-wave antennas," IEEE Transactions on Antennas and Propagation, vol. 57, no. 7, pp. 1894-1906, July 2009.

[5] D. Pozar, Microwave Engineering, 3rd ed. John Wiley and Sons, 2005

[6] C. Caloz and T. Itoh, "Array factor approach of leaky-wave antennas and application to 1-d/2-d composite right/left-handed (CRLH) structures," IEEE Microwave and Wireless Components Letters, vol. 14, no. 6, pp. 274-276, November 2004 